\title{
Sbiancare il cigno nero? Strategie e competenze manageriali per riconoscere il nuovo ${ }^{1}$
}

\author{
Maria Laura Frigotto - Alessandro Narduzzo
}

\begin{abstract}
Obiettivo del paper: La letteratura che analizza come le organizzazioni affrontano $i$ black swan tende a enfatizzare l'importanza di apprendere dall'esperienza, estendendo nel tempo il numero di casi che l'organizzazione è in grado di gestire. Questo paper propone un approccio radicalmente diverso e prevede che le organizzazioni possano affrontare gli eventi inattesi mentre si manifestano, invece di "sbiancarli" dopo che si sono manifestati. A tal fine le organizzazioni devono sviluppare un sistema di competenze che consenta loro di mettere in atto strategie differenti rispetto a quanto previsto dallapproccio tradizionale.

Metodologia: Il lavoro inquadra gli studi manageriali sulla gestione delle emergenze rispetto al problema dei black swan utilizzando come prospettiva di analisi lapproccio comportamentale alle organizzazioni elaborato dalla Scuola di Carnegie.

Risultati: La formulazione di due strategie e di un sistema di competenze che consentano al management di riconoscere e gestire gli eventi inattesi, senza ridurli ad eventi rischiosi.

Limiti della ricerca: Il presente lavoro propone una concettualizzazione dei problemi delle imprese che gestiscono black swan, senza distinguere le specificità delle diverse situazioni decisionali.

Implicazioni pratiche: Il paper identifica un sistema di competenze decisionali e organizzative, sviluppando le quali le imprese possono gestire situazioni black swan mentre accadono.

Originalità del paper: Il lavoro si distingue dalla prevalente letteratura manageriale perché elabora una concettualizzazione della gestione dei black swan coerente con la natura ontologica di questi fenomeni.
\end{abstract}

Parole chiave: eventi inattesi; black swan; gestione delle emergenze; preparazione.

Purpose of the paper: The literature that analyzes how organizations deals with black swans tends to emphasize the importance of learning from experience, extending over time the number of cases that the organization is able to manage. This paper proposes a radically different approach suggesting that organizations can face unexpected events as they occur, instead of "whitening" the black swans after they have occurred. To this end, organizations develop a peculiar system of capabilities and set of strategies.

1 Questo studio si è svolto nell'ambito del progetto di ricerca "Riconoscere e affrontare le nuove emergenze" finanziato dalla Provincia Autonoma di Bolzano (Secondo bando di concorso L.P. 14/2006) 
sinergie Vol. 34, N. 99, 2016

Methodology: This study reviews the managerial literature on black swans, along with the theoretical approach to organizations of the Carnegie School. The proposed theoretical framework is stimulated by a critical discussion of the literature on emergency management.

Findings: The formulation of two strategies and a system of competencies that allow to recognize and manage unexpected events without "transforming" them into risky events.

Research limitations: The original conceptualization of the management of black swans framed by this study does not distinguish the specificities of different contexts that may affect the decision-making process.

Research and managerial implications: The paper identifies a system of organizational capabilities that allow the firm to detect and manage black swan events while they happen.

Originality/value of the paper: This work develops a conceptualization of the management of black swan that is consistent with the ontological nature of these phenomena.

Key words: unexpected events; black swan; emergency management; preparedness.

\section{Introduzione}

Lo studio delle organizzazioni e del loro funzionamento si è tradizionalmente focalizzato sugli eventi ricorrenti e sulle situazioni che le organizzazioni si trovano ad affrontare più frequentemente, con l'obiettivo di migliorarne le performance di successo affinando e consolidando risposte efficaci ed efficienti. Recentemente, e in particolare la letteratura sull'apprendimento, si è rivolta allo studio di eventi unici (o a frequenza bassissima) e dalle conseguenze dirompenti dovute almeno in parte al fatto che le organizzazioni non sono preparate a gestirli, con una duplice motivazione. Da un lato, alcune manifestazioni di tale fattispecie rappresentano casi non più trascurabili rispetto all'opinione pubblica o all'esigenza di supportare chi si trova a doverli gestire (Tierney et al. 2001). L'attacco delle Torri Gemelle è un esempio emblematico di questi disastri inattesi, atipici ed estremamente gravi. Dall'altro, tali fenomeni, malgrado la loro estrema peculiarità, possono aprire ad una più profonda comprensione delle dinamiche decisionali e di apprendimento degli eventi ad alta frequenza, e che quindi riguardano la più vasta pletora delle organizzazioni impegnate nelle loro attività tipiche. Tali fenomeni sono stati definiti in vario modo nella letteratura, enfatizzando sfumature diverse intorno alla loro bassa frequenza e all'effetto sorpresa ad essi associato: "cigni neri" o black swan (Taleb, 2007), "eventi rari" (Lampel e Shapira 2001), "sorprese” (Pina e Cunha et al., 2006), l'“inatteso" (Weick e Sutcliffe, 2007), l' "impensabile” (Lagadec, 2006). Lambito empirico nel quale sono stati maggiormente studiati è quello delle emergenze. Tuttavia la letteratura di emergency managment si è mostrata poco ricettiva nel considerare la natura dei cigni neri, quali eventi inattesi e sostanzialmente sconosciuti, focalizzandosi invece sul perfezionamento del sistema di gestione delle emergenze in situazioni tipiche (Boin e 'T Hart, 2006; 
Lagadec, 2006) e al più "sbiancando" il cigno nero, ossia inserendolo nella lista delle situazioni tipiche.

Losservazione del comportamento dei decisori rispetto a tali eventi ha evidenziato che la resistenza a trattare i cigni neri per quello che sono costituisce una caratteristica della cognizione umana e non solo una posizione della letteratura. Le persone tendono a razionalizzare ex-post gli accadimenti inattesi, e sono soggetti al cosiddetto hindsight bias (Fischhoff, 1975; Harrison e March, 1984). In altre parole, esse tendono a sovrastimare la prevedibilità di un evento dopo che questo ha avuto luogo. Gli individui pongono l'accento sul fatto che l'informazione era disponibile, mentre trascurano le difficoltà che hanno incontrato a interpretare tale informazione come rivelatrice di ciò che sarebbe accaduto. "Le persone hanno l'impressione che i decisori avrebbero potuto sapere e, di conseguenza, avrebbero dovuto sapere, che cosa sarebbe accaduto" (Harrison e March, 1984, p. 411) e quindi avrebbero potuto e dovuto essere pronti ad affrontare tale scenario.

Questo lavoro inquadra il fenomeno del riconoscimento dei cigni neri da parte dei decisori, spiega quali sono gli ostacoli che il decisore incontra nel riconoscere un cigno nero in un contesto organizzativo, e individua due aspetti che influenzano la sua capacità decisionale, suggerendo come l'attenzione verso il cigno nero si possa tradurre in prassi manageriale, in modelli cognitivi per le decisioni, in sistemi di competenze manageriali per la percezione e la loro gestione.

\section{Riconoscere il nuovo: come affrontare un ossimoro}

La letteratura sul problem solving ha indagato, principalmente dal punto di vista del decisore individuale, il processo attraverso il quale una situazione viene inquadrata e formulata in termini di problema per poi essere risolta (Newell e Simon, 1972). Gli studi hanno tuttavia solo marginalmente considerato che non tutte le situazioni sono presentate e rappresentate come problemi da risolvere; il fatto stesso di riconoscere una situazione come problema da risolvere o meno costituisce un tema centrale nella comprensione della risoluzione dei problemi. I contributi su questo tema sono numericamente ancora limitati ${ }^{2}$ e concettualmente non offrono una prospettiva sistematica del fenomeno. Evidenza ne è il fatto che gli autori utilizzano concetti e locuzioni differenti (Cowan, 1986, p. 763): formulazione del problema (Lyles e Mitroff, 1980), riconoscimento del problema (Mintzberg et al., 1976), percezione del problema (Billings et al., 1980; Kiesler e Sproull, 1982), rilevamento del problema (Klein et al., 2005).

Seguendo i modelli teorici elaborati nell'ambito delle discipline manageriali (Hermann, 1972; Billings et al., 1980; Cowan, 1986), il riconoscimento di un problema si basa sul confronto tra lo stato atteso della realtà (generato in relazione alle aspirazioni) e la situazione percepita, per cui quando si percepisce una dissonanza tra realtà e aspettative e questo

2 Nella letteratura gli studi più citati che fanno esplicitamente riferimento a questo tema sono: Smith, 1989; Schön, 1983; Lyles e Mitroff, 1980; Mintzberg et al., 1976; Billings et al., 1980; Kiesler e Sproull, 1982; Klein et al., 2005. Non abbiamo riscontro di una pubblicazione di rassegna focalizzata su questa tematica.
Maria Laura Frigotto Alessandro Narduzzo Sbiancare il cigno nero? Strategie e competenze

manageriali per riconoscere il nuovo 
sinergie Vol. 34, N. 99, 2016

supera una soglia definita, un caso viene inquadrato come sufficientemente diverso (nuovo) e quindi problematico.

In questi modelli la presa di coscienza di essere di fronte ad un problema è inquadrata in una prospettiva generale, tuttavia essi trascurano di trattare in maniera critica la fase di percezione e interpretazione dei segnali. La principale conseguenza di questo approccio è che i modelli focalizzati sulle strategie di soluzione presentano una forte semplificazione di tutta la fase di riconoscimento, eventualmente rinviata a fasi precedenti. Primo, i modelli presentano il riconoscimento come un processo sequenziale e automatico, trascurandone la natura ricorsiva ${ }^{3}$. Secondo, considerano i segnali come delle manifestazioni neutre e inequivocabili dell'ambiente che "attendono di essere prese in considerazione" (Seidl, 2004, p. 156). Tuttavia, i segnali "non sono eventi primitivi - sono costruzioni generate da persone che cercano di capire le situazioni" (Klein et al., 2005, p. 17), quindi presuppongono un sistema di sensori che tuttavia è in grado di coglierne solo alcuni. Terzo, laccumulazione di discrepanze fino al raggiungimento di una soglia necessaria per scatenare l'identificazione della novità appare un costrutto poco convincente, che tra l'altro non è mai stato sottoposto ad un vaglio empirico ${ }^{4}$. L'importanza attribuita ai segnali dipende da chi li osserva; inoltre è plausibile contrapporre l'ipotesi che non sia la numerosità ma la salienza delle discrepanze a scatenare una revisione della percezione e delle categorizzazioni (Einhorn e Hogarth, 1981).

Infine, tali modelli presuppongono che sia attivo un sistema razionale di scansione continua dellambiente alla ricerca di indizi di anomalia e quindi di novità. Tale presupposto però si scontra con i limiti cognitivi manifestati dal decisore e con la dimensione organizzativa che supporta o inibisce la scansione dell'ambiente, aggravando o alleggerendo le difficoltà individuali, ed influenzando in definitiva aspettative e livelli di aspirazione.

La prospettiva di razionalità estetica di Shackle aggiunge a questo quadro che non solo lesplorazione della realtà avviene tipicamente entro i confini dei domini di conoscenza già in repertorio (Cohen e Levinthal, 1994), ma che anche l'immaginazione subisce questo limite. Infatti, gli individui mostrano una limitata capacità di immaginazione rispetto a ciò che non si è già sperimentato (Shackle, 1979): l'elicitazione di alternative immaginarie (imagined experiences) ritenute possibili è vincolata da ciò che si è già sperimentato. Questo contributo evidenzia che i cigni neri sono compresi e possono essere riconosciuti quando il loro timing è imprevisto, ma quando fanno parte della conoscenza già posseduta. Sono ossia eventi rari e inattesi, ma non nuovi. Nel caso in cui questa conoscenza sia andata persa nel tempo o non sia stata ancora generata, ossia gli eventi siano genuinamente nuovi, i cigni neri rappresentano una fattispecie

3 Per esempio, con riferimento al modello di Cowan (1986), la ricerca di informazioni caratterizza le fasi di categorizzazione e di diagnosi, mentre rappresenta un' attività ripetuta anche nella fase di ricerca di soluzioni.

4 Il modello di riferimento in questo caso è quello formulato da Cowan (1986) che non è basato su evidenze empiriche, né è stato successivamente validato (Klein et al., 2005). Al meglio della nostra conoscenza, avendo preso in rassegna la letteratura sull'identificazione dei nuovi problemi, il modello di Cowan (1986) manca ancora di un riscontro empirico. 
particolare, rispetto alla quale i modelli sopraccitati, che già evidenziano dei limiti nell'affrontare anche solo la rarità dei back swan, sono largamente inadeguati.

La prima difficoltà nel comprendere e modellizzare un sistema organizzativo adeguato a questo caso consiste nel rappresentare in forma generale e non contraddittoria quello che nel linguaggio naturale assume la forma di un ossimoro: ri-conoscere qualcosa che non si è ancora conosciuto.

\section{Le nuove emergenze e il concetto di preparedness negli studi sulla gestione delle emergenze}

Un contesto empirico che offre indicazioni particolarmente rilevanti per affrontare l'ossimoro introdotto nella precedente sezione è quello delle emergenze, poiché, in questo ambito, convivono confusi sotto la stessa etichetta black swan gli eventi rari, le sorprese e le novità.

Infatti, molte delle crisi più gravi degli ultimi vent'anni sembrano differire sostanzialmente dai casi che hanno dato vita agli studi di emergency management nel 1950 e il 1960: dagli attacchi terroristici dell'11 settembre 2001 alla contaminazione da encefalopatia spongiforme bovina (cosiddetta "mucca pazza"), al blackout nell'Europa continentale del 2006 o all'uragano Katrina. Contrariamente a quanto accade per le emergenze di tipo "tradizionale", le nuove emergenze sono "incredibili", "impensabili" e "inconcepibili" prima che abbiano effettivamente luogo (Lagadec, 2006). Tali emergenze appaiono sempre più come "not-textbook events" (Lagadec, 2006, p. 489) e riflettono i problemi del mondo moderno (di tipo sociale, politico, legato alle risorse naturali) per cui si manifestano come "nuove".

Alcuni studiosi hanno ritenuto tali emergenze archetipiche di una categoria distinta, le "nuove emergenze", non solo perché presentano caratteristiche difformi da quelle solitamente prese in esame negli studi di emergency management, ma anche e soprattutto perché non vengono adeguatamente inquadrate e affrontate ricorrendo ai tradizionali sistemi operativi di gestione dell'emergenza (Boin e 'T Hart, 2006, p. 4; Lagadec, $2006)^{5}$. Esse rappresentano una categoria aperta, la cui popolazione prende forma gradualmente, solo dopo essere divenuta realtà. Questo rende difficile prevedere le nuove emergenze, così come ragionare su emergenze tipiche rispetto alle quali attivare risposte standardizzate. Tradizionalmente, le organizzazioni per la previsione e la gestione delle emergenze fondano

5 Malgrado ogni emergenza, anche quelle che meglio aderiscono a categorie standard, presenti caratteristiche specifiche del contesto tali da renderla diversa dalle precedenti, gli eventi identificati nuove emergenze sono significativamente diversi dai precedenti nel senso che richiedono una revisione delle categorie usate per afferrarli e delle pratiche in uso per gestirli. È difficile indicare eventi storici come esempi puri di nuove emergenze, da un lato poiché la realtà mal si adatta ad essere rappresentata in una sola dimensione, dall'altro poiché il nuovo è un concetto relativo (cambia a seconda del soggetto che lo valuta, dello spazio e del tempo) che necessita del confronto con la conoscenza esistente per essere compreso e individuato. Il concetto di "nuova emergenza" è utile poiché consente di evidenziare non solo il carattere raro o sorprendente degli eventi indicati come cigni neri, ma il loro carattere inedito e sconosciuto prima della loro manifestazione.
Maria Laura Frigotto

Sbiancare il cigno nero?

Strategie e competenze manageriali per riconoscere il nuovo 
sinergie Vol. 34, N. 99, 2016

e orientano la propria azione sul concetto di preparedness che è stato teorizzato dalla letteratura dedicata (Quarantelli, 1988; Alexander, 2002; Coppola, 2011) con l'obiettivo di migliorare la risposta ad una lista chiusa (e idealmente completa) di emergenze conosciute e prevedibili su base probabilistica. Le nuove emergenze impongono di rivedere quest'idea di preparedness e di ridiscuterne il significato. Ciò richiede un esercizio di pensiero innovativo che va oltre il miglioramento marginale ottenuto aumentando lo spettro delle contingenze del rischio (Quarantelli et al., 2006, p. 36). Questo passaggio richiama una discontinuità analoga al salto tra single e double loop learning (Argyris e Schön, 1978) ${ }^{6}$. Mentre il single loop learning rimanda a una apprendimento nel senso dell'incremento di contingenze che lorganizzazione è in grado di affrontare, il double loop learning richiede di mettere in discussione il modo con cui si apprende.

Le conseguenze prodotte dall'incapacità di riconcettualizzare il tema della preparedness rispetto alle emergenze di tipo inatteso sono facilmente intuibili: le analisi e i tentativi di apprendimento che seguono una nuova emergenza sono per la maggior parte rivolti a correggere quelle che sono viste come deficienze contingenti (ad esempio, nella tecnologia, nella struttura organizzativa, nella cultura organizzativa, ecc.) piuttosto che come errori connaturati al tradizionale concetto di preparedness.

Un esempio della difficoltà a superare una concezione tradizionale di preparedness è illustrato molto bene dalla reazione che il governo USA e le organizzazioni responsabili della gestione delle emergenze hanno avuto a seguito degli eventi dell'11 settembre 2001. Milioni di dollari sono stati stanziati per comprendere gli errori commessi nella gestione dellemergenza e per definire un piano di emergency management adatto ad affrontare situazioni analoghe. Nel 2004 è stato implementato un nuovo sistema per la gestione delle emergenze il National Incident Management System (NIMS) che prevede a livello federale una piattaforma di risorse, attività e strutture organizzative standardizzate da combinare in maniera flessibile di fronte alle emergenze. Nel 2006 è stata la volta dei FEMA Principles of Emergency management che prescrivono come gestire attività critiche come lo scambio di informazioni o la definizione del controllo gerarchico. Nessuno di questi sistemi prevede però che l'emergenza possa essere di tipo anomalo o nuovo, né sono definite delle procedure per la comprensione di situazioni nuove o inattese. Nel 2005, di fronte al New Terrorism Response Plan per la città di New York, il capo dei vigili del fuoco, Peter Hayden, ha dichiarato che, nonostante gli sforzi e le ingenti risorse, questa "ricetta per i disastri" non lascia la città meglio preparata ad un attacco terroristico di quanto non fosse l'11 settembre ${ }^{7}$. 


\section{Ostacoli al riconoscimento}

\subsection{La capacità di riconoscere}

manageriali per

Il tema del riconoscimento delle situazioni inattese, come ad esempio le nuove emergenze o i cigni neri, può essere affrontato da diversi punti di vista, assumendo come explanans principale le tecnologie della comunicazione, la cultura e la motivazione del personale o altro (Quarantelli, 1988; Turner, 1976). Rispetto a queste possibilità, sembra appropriato proporre una prospettiva integrata avente come oggetto di analisi l'organizzazione, nella quale fattori interni ed esterni si combinano e interagiscono.

Nella tradizione della scuola di Carnegie ${ }^{8}$ (Gavetti et al., 2007, p. 523), assumiamo il processo decisionale come locus privilegiato per studiare le organizzazioni, vista la loro centralità sia sostantiva che procedurale nello scatenare l'azione e nel perseguire i risultati organizzativi (Simon, 1947). La fase del processo decisionale su cui ci focalizziamo è quella in cui i cigni neri vengono riconosciuti e gestiti, ed è caratterizzata da aspetti cognitivi, sociali, organizzativi, nonché tecnologici. La prospettiva organizzativa consente di analizzare a diversi livelli e nel loro insieme, le determinanti e dinamiche di tale processo.

La ricerca sui disastri (Turner, 1976), sulle crisi organizzative (Sheaffer et al., 1998) e gli studi sugli errori (Ramanujam, 2003) hanno evidenziato che il fallimento è legato all'insuccesso nel cogliere e rispondere ai cosiddetti incubatori (Turner, 1976), precursori (Tamuz, 2004), indizi o segnali deboli (Haeckel, 2004; Rerup, 2009), anomalie (Weick et al., 1999) che rivelano minacce future (Vaughan, 1996; Weick e Sutcliffe, 2007), mentre le organizzazioni che meglio prevengono le crisi, investono nella capacità di riconoscere tali segnali (Marcus e Nichols, 1999; Haeckel, 2004; Dillon e Tinsley, 2008). Definiamo la capacità di riconoscimento come la capacità di dedicare attenzione organizzativa al nuovo ${ }^{9}$, ossia la capacità di notare, codificare, mettendo in costante discussione sia le istanze, e quindi il repertorio di categorie a disposizione per dare senso al mondo (ad esempio emergenza/non emergenza), sia le risposte, ossia il repertorio di azioni alternative a disposizione (ad esempio routine, progetti, programmi e procedure). La capacità di riconoscimento è una competenza organizzativa e come tale è parte di un più ampio sistema di competenze e di risorse dell'organizzazione, è dipendente dal contesto ed è suscettibile di apprendimento, sviluppo e adattamento (Narduzzo e Warglien, 1998).

8 La Carnegie School ha avuto origine a partire dai contributi di Cyert e March (1963), March e Simon (Organizations, 1958) e Simon (1947).

9 Per formulare questo concetto ci rifacciamo alla nozione di organizational attention di Ocasio (1997) definita come "the noticing, encoding, interpreting, and focusing of time and effort by organizational decision-makers on both (a) issues: the available repertoire of categories for making sense of the environment; problems, opportunities and threats; and (b) answers: the available repertoire of action alternatives; proposals, routines, projects, programs, and procedures" ( $\mathrm{p}$. 189). 


\section{sinergie}

Vol. 34, N. 99, 2016

\subsection{I limiti della razionalità decisionale}

La difficoltà di riconoscimento delle situazioni inattese rimanda a diversi tipi di limiti che riguardano direttamente l'attore decisionale. Innanzitutto, le aspettative del decisore sono incomplete, rispetto ai possibili stati del mondo o alle probabilità con cui questi si possono verificare. Inoltre il decisore agisce in condizioni di razionalità limitata che riguardano a) le limitate capacità di computazione e di memoria, b) la saturazione dellattenzione, specie in presenza di improvvisi ed elevati flussi di informazione (March e Simon, 1958; Cyert e March, 1963; Ocasio, 1997), c) la gestione delle priorità, soprattutto nelle situazioni in cui problemi semplici e complessi debbano essere gestiti contemporaneamente.

Da questo punto di vista la letteratura organizzativa sulle emergenze offre indicazioni rilevanti per comprendere alcune delle difficoltà che $\mathrm{i}$ decisori incontrano nell'identificare situazioni inattese. Turner and Pidgeon (1997, p. 52) osservano che "le persone sono inibite dal comunicare un problema [...] perché la loro attenzione è completamente occupata nel trattare problemi più chiaramente definiti”. Inoltre, Rudolph e Repenning (2002) hanno evidenziato che il flusso d'informazioni e la quantità di compiti da svolgere nell'unità di tempo in contesti ad alta posta in gioco $\mathrm{e}$ ad alta volatilità influenza in modo determinante l'efficacia del processo di gestione dell'emergenza. Le persone sbagliano di fronte a nuove situazioni problematiche che impongano vincoli di tempo e risultato (Weick, 1990; Weick, 1993).

La letteratura comportamentale ha chiarito che i decisori tendono a trascurare anomalie o variazioni rispetto ai casi tipici rivelandosi vittime di bias cognitivi o trappole di competenza (Levitt e March, 1988). I decisori prestano maggiore attenzione a informazioni e dettagli che si manifestano in modo caratteristico nelle situazioni standard e trascurano aspetti nuovi, differenti o anche solo diversi dallordinario. In altre parole, i decisori tendono a semplificare l'interpretazione del mondo (Turner e Pidgeon, 1997), il che consente loro di reagire ad una situazione attivando la risposta standard, per potersi dedicare al task successivo. Questa strategia si basa sul presupposto implicito che una volta che una crisi è stata identificata, questa può essere gestita e controllata poiché esiste un pacchetto efficace di attività, strumenti, procedure da seguire (Lagadec, 2006, p. 496). In questo modo, tuttavia, accade che le anomalie siano ignorate, tendano ad accumularsi e le conseguenze indesiderate siano prese in considerazione solo quando possono essere difficilmente contenute (Weick et al., 1999).

Infine, uno degli aspetti solitamente trascurati nell'approccio razionale riguarda il ruolo delle emozioni che invece nella gestione delle emergenze, sia per la elevata posta in gioco (vite umane, capitali, risorse naturali) che per il carattere improvviso (sorpresa e disorientamento) con cui si manifestano (Pina e Cunha et al., 2006), risulta di primaria importanza. Nel caso della gestione dell'emergenza verificatasi l'11 settembre 2001 a New York, Frigotto e Narduzzo (2010) hanno studiato il comportamento degli operatori del centralino 911 che hanno gestito le chiamate di emergenza nel periodo intercorso tra l'attacco al primo grattacielo e il crollo del secondo grattacielo. La presa di coscienza da parte degli operatori del 911 
che per alcuni chiamanti non vi fossero via di fuga e che fossero destinati a soccombere per il fumo, ha prodotto stati di coinvolgimento emotivo tali da rendere più difficoltoso o addirittura da arrestare il processo di riconoscimento dell'emergenza e dell'evoluzione della situazione.

\subsection{Il contesto organizzativo}

Un assunto fondamentale dello studio delle decisioni secondo la prospettiva della scuola di Carnegie è che la cognizione umana, che avviene in contesti sociali o in contesti sociali organizzati, non sia riducibile alla cognizione individuale, (Simon, 1947). Hutchins (1995) ha mostrato che la capacità cognitiva dellorganizzazione e degli individui si dispiega ed è forgiata da pratiche, regole, procedure, risorse, tecnologie esistenti, che riflettono la cultura e la struttura sociale delle organizzazioni.

Il fattore organizzativo forse più semplice da considerare è la divisione del lavoro per cui il riconoscimento e l'elaborazione di stimoli e informazioni, così come la produzione di conoscenza rispetto ad una situazione, non sono processi centralizzati, bensì distribuiti (von Hayek, 1937). Attori diversi (individui o parti delle organizzazioni) raccolgono informazioni, trattengono e trasmettono frammenti di esse che vengono usate per prendere decisioni da altri attori (Persico, 2014).

La natura distribuita e frammentaria dei processi di percezione e di identificazione di stimoli e informazioni presenta alcune importanti implicazioni. Primo, i sensori del sistema tendono ad essere specializzati, non dispongono di una conoscenza generale della situazione e quindi non sono in grado di produrre una lettura completa dei fenomeni (Winter, 2004). La capacità di riconoscimento è disomogenea nell'organizzazione e tipicamente è maggiore in prossimità della manifestazione delle informazioni ossia a livello locale (Lampel e Shapira, 2001).

Secondo, i problemi di trasmissione delle informazioni e di comunicazione influenzano significativamente la capacità organizzativa di riconoscere situazioni inattese. Rientrano in questa categoria sia i banali errori o i ritardi di trasmissione (ad esempio le informazioni sono inviate al destinatario sbagliato o con il contenuto sbagliato), così come le situazioni più complesse in cui l'informazione è ambigua, la percezione è problematica e di conseguenza la comunicazione è imprecisa.

Terzo, la divisione del lavoro e la natura delle interdipendenze tra le unità organizzative influenza la capacità di un sistema cognitivo distribuito di collegare tra di loro stimoli e informazioni. Da questo punto di vista l'architettura del sistema di comunicazione (ad esempio il numero di passaggi delle informazioni o la presenza di ridondanza della comunicazione) condiziona in modo decisivo la capacità di trasmettere ed elaborare in modo distribuito i segnali (Bavelas, 1950).

Un altro fattore organizzativo certamente rilevante rispetto alla capacità di riconoscimento di situazioni nuove e inattese sono le relazioni sociali all'interno dell'organizzazione. Le relazioni sociali sono influenzate da numerosi elementi quali la dimensione gerarchica (capo/collaboratore), lo stile di leadership, la cultura organizzativa, ma anche gli atteggiamenti spontanei delle persone che entrano in relazione (simpatia/antipatia,
Maria Laura Frigotto
Alessandro Narduzzo Sbiancare il cigno nero? Strategie e competenze manageriali per riconoscere il nuovo 
sinergie Vol. 34, N. 99, 2016

reverenza/arroganza, ecc.), e le usanze informali che definiscono ciò che è socialmente accettabile. Ad esempio, Turner e Pidgeon (1997) hanno riscontrato una elevata tolleranza da parte delle organizzazioni che gestiscono le emergenze rispetto al mancato rispetto di piani di intervento considerati obsoleti. Il tipico fenomeno del groupthink descritto da Janis (1982) rappresenta il risultato di una cultura della coesione e del consenso portata allestremo, per cui nei gruppi non vengono valutate soluzioni alternative né aperta una discussione critica. La cultura del successo diffusa in NASA è uno degli elementi che ha condotto al disastro del Challenger (Vaughan, 1996). Tale aspetto culturale si combina con dinamiche di potere gerarchico nel caso del disastro aereo a Tenerife studiato da Weick (1990), e, più in generale, con attribuzioni di potere, risorse o motivazione, poiché spesso chi ha le informazioni non può o non è incentivato a sollevare dubbi, problemi e ad intervenire (Rerup, 2009).

\subsection{L'inerzia delle procedure operative standard}

Nell'emergency management l'idea di che le competenze per gestire le emergenze organizzative come repertorio di procedure efficienti trova espressione nella logica della preparedness tradizionalmente intesa.

Le procedure sono il prodotto della natura adattiva delle organizzazioni (Cyert e March, 1963; Nelson e Winter, 1982) che tendono a concentrarsi sulla risposta ai problemi che si presentano come maggiormente rilevanti. Tuttavia, le organizzazioni tendono anche a focalizzarsi su ciò che hanno appreso e che riescono a fare meglio, e a considerare gli stimoli esterni come istanze che richiedono la loro competenza. La conseguenza di ciò è che il sistema di competenze acquisito in situazioni di emergenza note inibisce la capacità di riconoscere le nuove emergenze che richiede un’apertura verso il nuovo, implica la disponibilità a sperimentare, presuppone unattenzione continua e una ricezione di informazioni che va continuamente stimolata e supportata per evitare che si fossilizzi in attività routinarie automatiche (Rerup, 2009).

Quando le organizzazioni per la gestione delle emergenze tendono a concentrarsi principalmente sullaffinamento delle pratiche già sperimentate e collaudate si espongono al rischio di restare intrappolate nel proprio sistema di competenze (Levitt and March, 1988) e rischiano di non accorgersi se e quando si trovano di fronte a situazioni nuove che richiedono di essere inquadrate e affrontate in modo differente (Tierney et al., 2001, p. 118).

\section{Strategie per affrontare le nuove emergenze}

\subsection{Mantenere l'attenzione e categorizzare i segnali deboli}

È importante chiarire che la letteratura è ben lungi dalla sistematizzazione della fattispecie e offre una conoscenza frammentata tra diversi approcci: Normal accidents (Perrow, 1999), Sensemaking (Weick, 1993), High Reliability Organizations (La Porte e Consolini, 1991; Bigley 
e Roberts, 2001). Tuttavia è possibile organizzare i contributi secondo due principali strategie per la gestione dei black swan. Nessuna di queste strategie cerca di negare la natura degli eventi black swan o propone di assimilarli a situazioni tradizionali.

La prima strategia prende in esame le condizioni per creare un disegno (struttura e processo) e una cultura organizzativa capaci di mantenere e potenziare l'attenzione verso i black swan.

Per quel che riguarda il disegno organizzativo, Rerup (2009) ha mostrato l'importanza di disporre di una rete di coordinamento e di comunicazione per la segnalazione delle anomalie sia lungo la gerarchia che al di fuori di essa. Lautore ha evidenziato che la capacità di riconoscimento è stata perseguita attraverso l'istituzione di gruppi di staff che al di fuori della linea gerarchica fungono da facilitatori nel far emergere potenziali minacce notate a diversi livelli dell'organizzazione. In questo modo, è stato ridotto il filtro che l'informazione subisce lungo la gerarchia a favore di un trasferimento maggiormente ricco di istanze e di dettagli. Weick et al. (1999) hanno osservato che nelle organizzazioni caratterizzate da elevata resilienza, quando gli eventi diventano critici e superano i confini dell'operatività standard, il controllo non è assunto da chi copre la posizione più alta nella gerarchia, ma da chi ha la maggiore expertise nel problema specifico. Nel caso delle portaerei, ad esempio, la localizzazione dei soggetti esperti avviene attraverso l'uso di "reti epistemiche" che mappano le competenze specifiche dei membri dellorganizzazione che si raggruppano sulla base del problema specifico. Questi team, come organizzazioni effimere (Lanzara, 1983) o sintetiche (Thompson, 1967), si dissolvono una volta che il problema è risolto. Lo studio di Oliver e Roos (2003) ha evidenziato l'importanza della prossimità fisica di chi lavora alla scansione continua dell'ambiente alla scoperta del nuovo, primo, come supporto necessario all'interpretazione del mondo e alla categorizzazione secondo o al di fuori delle categorie organizzative esistenti.

In termini di processo, al fine di amplificare la capacità organizzativa di riconoscere il nuovo, è possibile provocare e stimolare un'azione tempestiva per osservare se questa produce effetti sorprendenti o anomali. In questo modo la percezione del nuovo è la conseguenza di una costruzione retrospettiva di significato (Weick et al., 1999): l'agire, l'esplorare sono il presupposto necessario per comprenderel'ambiente. In contesti di emergenza, l'improvvisazione (Kendra e Wachtendorf, 2006; Trainor, 2004) e il bricolage (Bechky e Okhuysen, 2011) sono stata indicate come efficaci soluzioni di risposta adattiva. In altri casi, queste soluzioni costituiscono una strategia temporanea di risposta rapida che consente di tamponare l'emergenza e di portare a conclusione un processo di interpretazione complesso che richiede tempo, al termine del quale attivare una risposta maggiormente adeguata al caso specifico (Chen et al., 2008; Faraj e Xiao, 2006).

Per quel che riguarda la cultura, fin dal contributo seminale di Turner (1976) si è compresa l'importanza che all'interno dellorganizzazione, ad ogni livello, sia possibile parlare apertamente e porre questioni, anche delicate, come quelle che riguardano l'azione dei superiori o di altre persone. Inoltre Weick et al. (1999) hanno riscontrato l'effetto positivo prodotto da una diffusa preoccupazione per il rischio di fallimento, che tiene alto
Maria Laura Frigotto Sbiancare il cigno nero? Strategie e competenze manageriali per riconoscere il nuovo 
sinergie Vol. 34, N. 99, 2016

il senso di responsabilità e la percezione dellimportanza del contributo dei singoli. Questi tratti, che possono essere diffusi tra gli individui attraverso una cultura organizzativa adeguatamente sostenuta, possono più semplicemente essere trovate negli individui stessi, come caratteristica dell'identità che essi hanno o che si costruiscono nell'organizzazione. L'identità infatti è qualcosa che costituisce gli individui, mentre la cultura è una condizione di contesto nella quale essi si trovano e al più è qualcosa che gli appartiene. Oliver e Roos (2003) hanno mostrato l'efficacia di dedicare tempo alla costruzione dell'identità dei gruppi: discutere su loro stessi, sugli scopi che li muovono, sul tipo di gruppo che vogliono diventare. Le identità fungono, infatti, da generatori di principi di azione impliciti, per cui per esempio appartenere al gruppo degli "smanettoni informatici" vuol dire chiudersi ore in solitudine al computer incuranti delle norme sociali o organizzative - come l'orario di lavoro - (Frigotto et al., 2013). Il riferimento a principi generali di comportamento risulta particolarmente efficace poiché questi costituiscono orientano l'azione e l'interpretazione anche in momenti di emergenza quando i sistemi di governo e di coordinamento, basati su regole o sull'autorità gerarchica, possono entrare in crisi (Dutton e Dukerich, 1991).

\subsection{Apprendere e prepararsi alle nuove emergenze}

La seconda strategia prende in esame le occasioni di apprendimento dalle nuove emergenze o da eventi che pur non essendo così dirompenti mostrano un elevato grado di novità rispetto allo standard. Questi casi comprendono anche i cosiddetti "quasi disastri" o "quasi incidenti".

Per quel che attiene alle strategie di apprendimento, la letteratura ha da tempo evidenziato la diffusa pratica di generazione di storie in corrispondenza di errori che sono stati commessi, chiamate anche post-mortem, al fine di ricostruire la catena causale che li ha provocati e di intervenire su tale dinamica in futuro (Weick et al., 1999). Nella letteratura e nella prassi consolidata questo approccio è proposto per migliorare l'efficienza operativa, prevenire il ripetersi di errori, risolvere problemi strutturali, favorire la circolazione della conoscenza. Nella nostra prospettiva, la produzione di storie costituisce una strategia importante per prepararsi al nuovo e alle nuove emergenze per una serie di ragioni.

Primo, le storie aiutano a costruire lidentità delle organizzazioni, dei gruppi e degli individui (Brown, 2001), ossia a definire un modo di essere, che in maniera ancora più generale dei principi, definiscono come i membri dellorganizzazione sono chiamati ad agire.

Secondo, le storie rappresentano il principale canale per generare e diffondere conoscenza sul nuovo e capacità per affrontarlo. Danno contenuto alle pratiche, anche a quelle in disuso, e trasmettono la saggezza oltre che l'atteggiamento emotivo con cui approcciare i problemi (Oliver e Roos, 2003). Consentono di definire rappresentazioni verosimili nelle quali pensare di situare l'azione. Per esempio quando i piloti dell'aeronautica militare italiana studiano le missioni parlano di picture della situazione (Frigotto e Zamarian, 2015). La letteratura sulla situated action ha mostrato 
come tale pratica infonda sicurezza e renda la risposta più rapida (Suchman, 1987).

Terzo, attraverso l'esercizio di generazione di storie plurime le organizzazioni espandono lo spettro delle loro aspettative sfruttando una realtà virtuale che loro stessi creano (Frigotto e Zamarian, 2010).

Dato che l'esperienza ripetuta di eventi simili, condizione necessaria all'apprendimento, non è possibile quando si parla di nuove emergenze, March et al. (1991) hanno argomentato che le organizzazioni "arricchiscono" le esperienze uniche che hanno a disposizione tentando di estrarvi più conoscenza rispetto al normale. Ciò avviene in tre modi. Le organizzazioni costruiscono "quasi storie" (near histories) nelle quali si esercitano a ipotizzare come sarebbero andati gli eventi cambiando alcune circostanze. In questo modo, comprendono da che cosa potrebbero derivare i disastri e imparano a che cosa prestare attenzione. Per esempio, i piloti dell'aeronautica studiati da Haunschild e Sullivan (2002) Morris e Moore (2000) e Frigotto e Zamarian (2010) ragionavano sugli "incidenti sicuri", ossia eventi che in circostanze leggermente diverse sarebbero sfociate in incidenti. Inoltre, le organizzazioni generano storie ipotetiche (hypothetical histories), che prendono spunto da un insieme di esperienze, e che fungono da matrice per generare una serie di storie modificando via via alcuni aspetti. Infine, raccolgono numerose storie redatte da soggetti diversi per competenze, ruoli, coinvolgimento, e che tuttavia riguardano lo stesso evento. In questo modo estendono la conoscenza delle problematiche affrontate ai diversi livelli e si moltiplicano le prospettive con cui guardare all'esperienza.

\section{Black swan e imprese: implicazioni manageriali}

Gli studi compiuti sulle organizzazioni che affrontano nuove emergenze offrono importanti indicazioni sulle possibili soluzioni organizzative e manageriali a disposizione delle imprese che vogliano acquisire e sviluppare competenze per affrontare eventi ontologicamente analoghi. Infatti, le situazioni di black swan che abbiamo descritto con riferimento alle organizzazioni di gestione dell'emergenza si presentano anche ad altre organizzazioni e imprese in contesti di business (Catino 2009). Per esempio, Kodak (Gavetti et al., 2005), Polaroid (Tripsas e Gavetti, 2000) e Facit (Starbuck, 1983) sono casi di black swan nel senso che i decisori chiave non hanno saputo riconoscere la novità tecnologica, competitiva e di mercato che si poneva di fronte a loro.

La sfida che le nuove emergenze e questi black swan pongono alle organizzazioni consiste nello sviluppare le competenze per riconoscere e reagire a eventi nuovi e inattesi e per i quali va elaborata una risposta adeguata. In una logica manageriale le competenze critiche dellorganizzazione non possono limitarsi alla capacità di risposta rapida ed efficace a un insieme chiuso di situazioni che viene via via aggiornato di fronte all'ultimo nuovo evento, ma devono essere estese alla capacità di riconoscere la prossima novità. Questa capacità consente di riconoscere e comprendere la novità nel momento stesso in cui sta divenendo realtà e l'expertise delle organizzazioni
Maria Laura Frigotto

Alessandro Narduzzo

Sbiancare il cigno nero?

Strategie e competenze

manageriali per

riconoscere il nuovo 
sinergie Vol. 34, N. 99, 2016

non consiste più nel raffinare una risposta efficiente rispetto a un numero finito di emergenze, ben identificate e identificabili.

Le due strategie delineate in questo paper sono funzionali al riconoscere a affrontare black swan e possono essere valide per tutte le organizzazioni: la prima, mantenere l'attenzione e categorizzare i segnali deboli, la seconda, attivare processi di apprendimento ad hoc. La loro realizzazione richiede lo sviluppo di competenze specifiche che operano nella direzione opposta a quella normalmente perseguita nelle organizzazioni: esse mirano all'esplorazione dello spazio del problema piuttosto che alladozione della soluzione più efficiente. Rispetto alla prima strategia, le competenze riguardano la capacità di progettare e gestire organizzazioni che siano resilienti, che sappiano valorizzare i segnali deboli e le expertise distribuite, che riescano ad adattarsi attraverso l'improvvisazione, e che siano in grado di apprendere dagli errori. Rispetto alla seconda strategia, è necessario sviluppare la capacità di lettura della realtà da diversi punti di vista, di immaginazione e di rielaborazione dellesperienza vissuta.

\section{Conclusioni}

I cosiddetti cigni neri rappresentano attualmente sia in letteratura che sul campo, eventi difficili da comprendere e da gestire rispetto ai quali il disegno organizzativo e le forme di apprendimento non hanno saputo offrire risposte adeguate alla natura del fenomeno. Tipicamente infatti, i cigni neri passano inosservati, fino al punto in cui eventualmente manifestano i propri effetti, spesso purtroppo tragici, come nel caso dei disastri. L'apprendimento inoltre, anche in seguito a queste esperienze, si concretizza nell'estensione del numero di situazioni che l'organizzazione monitora ed è in grado di gestire, ma non si traduce in una maggiore preparazione a cogliere ed affrontare il prossimo cigno nero. Questa tendenza riguarda le organizzazioni in generale a causa delle caratteristiche del comportamento individuale, sociale e organizzativo che abbiamo evidenziato assumendo il punto di vista della Scuola di Carnegie.

Considerare seriamente la natura dei black swan, in quanto eventi nuovi, seppur per diverso grado, ha imposto di considerare che le organizzazioni si trovano ad affrontarli in una situazione di assenza di esperienza e di conoscenza riguardo la loro forma e la possibilità della loro esistenza. Tali elementi costituiscono invece tradizionalmente l'elemento necessario per riconoscerli e gestirli.

La soluzione che abbiamo proposto in questo articolo non riguarda quindi la creazione di conoscenza riguardo i black swan che si sono manifestati, cosa che equivale a trasformarne la natura da eventi nuovi e eventi conosciuti, o in altri termini a "sbiancare il cigno nero", quanto a costruire conoscenza riguardo il nuovo e ciò che, pur non conosciuto, si manifesta. A tal fine le organizzazioni possono sviluppare un disegno organizzativo e un sistema di competenze aperto a ridiscutere le proprie categorizzazioni del mondo e le relative premesse.

In questo lavoro abbiamo proposto una concettualizzazione dei problemi delle imprese che gestiscono black swan, che non distingue 
le specificità delle diverse situazioni decisionali. Riteniamo che questa limitazione costituisca una possibile linea di approfondimento per la ricerca futura.

\section{Bibliografia}

ALEXANDER D.E. (2002), Principles of Emergency Planning And Management, Oxford University Press, Edinburgh, UK.

ARGYRIS C., SCHÖN D. (1978), Organizational Learning: A Theory of Action Perspective, Addison-Wesley, Reading, MA.

BAVELAS A. (1950), "Communication Patterns in Task-Oriented Groups", The Journal of the Acoustical Society of America, vol. 22, n. 6, pp. 725-730.

BECHKY B.A., OKHUYSEN G.A. (2011), "Expecting the Unexpected? How Swat Officers and Film Crews Handle Surprises", Academy of Management Journal, vol. 54, n. 2, pp. 239-261.

BIGLEY G.A., ROBERTS K.H. (2001), “The Incident Command System: HighReliability Organizing for Complex and Volatile Task Environments", The Academy of Management Journal, vol. 44, n. 6, pp. 1281-1299.

BILLINGS R.S., MILBURN T.W., SCHAALMAN M.L. (1980), "A model of crisis perception: a theoretical and empirical analysis", Administrative Science Quarterly, vol. 25, n. 2, pp. 300-316.

BOIN A., 'T HART P. (2006), “The crisis approach”, in Quarantelli E.L., Dynes R., Rodriguez H. (a cura di), Handbook of Disaster Management, Springer, New York, pp. 42-54.

BOUQUET C., BIRKINSHAW J. (2008), "Weight versus voice: How foreign subsidiaries gain attention from corporate headquarters", Academy of Management Journal, vol. 51, n. 3, pp. 577-601.

BROWN A. (2001), "Organizational studies and identity: Towards a research agenda”, Human Relations, vol. 54, n. 1, pp. 113-21.

CATINO M. (2009), Miopia organizzativa. Problemi di razionalità e previsione nelle organizzazioni, Il Mulino, Bologna.

CHEN R., SHARMAN R., RAO H.R., UPADHYAYA S.J. (2008), "Coordination in emergency response management", Communications of the ACM, vol. 51, n. 5, pp. 66-73.

COHEN W.M., LEVINTHAL D.A. (1994), "Fortune Favors the Prepared Firm", Management Science, vol. 40, n. 2, pp. 227-251.

COPPOLA D.P. (2011), International Disaster Management, ButterworthHeinemann, Burlington, MA.

COWAN D.A. (1986), "Developing a process model of problem recognition”, The Academy of Management Review, vol. 11, n. 4, pp. 763-776.

CYERT R.M., MARCH J.G. (1963), A behavioral theory of the firm, Prentice-Hall, Englewood Cliffs, NJ.

DILLON R.L., TINSLEY C.H. (2008), "How near-misses influence decision making under risk: A missed opportunity for learning", Management Science, vol. 54, n. 8 , pp. $1425-1440$.

DUTTON J., DUKERICH J. (1991), "Keeping an eye on the mirror: Image and identity in organizational adaptation", Academy of Management Journal, vol. 34, n. 3, pp. 517-554.
Maria Laura Frigotto

Sbiancare il cigno nero?

Strategie e competenze

manageriali per

riconoscere il nuovo 
sinergie Vol. 34, N. 99, 2016

EINHORN H.J., HOGARTH R.M. (1981). "Behavioral decision theory: Processes of judgment and choice", Journal of Accounting Research, vol. 19, n. 1, pp. $1-31$.

FARAJ S., XIAO Y. (2006), "Coordination in fast-response organizations", Management Science, vol. 52, n. 8, pp. 1155-1169.

FISCHHOFF B. (1975), "Hindsight is not equal to foresight: The effect of outcome knowledge on judgment under uncertainty", Journal of Experimental Psychology, Human Perception and Performance, vol. 1, n. 3, pp. 288-299.

FRIGOTTO M.L., COLLER G., COLLINI P. (2013), “The relationship between strategy and management control systems as an emerging dynamic process", Journal of Management and Governance, vol. 17, n. 3, pp. 631-656.

FRIGOTTO M.L., NARDUZZO A. (2010), "9/11: organizations coping with the unexpected”, paper presentato all' EGOS Colloquium, Bergen, Norway.

FRIGOTTO M.L., ZAMARIAN M. (2010), "Staying aloft: resiliency and cost effectiveness in facing complex environments", paper presentato al $10^{\circ}$ Workshop di Organizzazione Aziendale - Bologna, 16-18 giugno 2010.

FRIGOTTO M.L., ZAMARIAN M. (2015) "Mindful by routine: evidence from the Italian Air Force Tornado crews flying practices", Journal of Management and Organization, DOI:10.1017/jmo.2014.85.

GAVETTI G., LEVINTHAL D., OCASIO W. (2007), "Perspective-Neo-Carnegie: The Carnegie School's Past, Present, and Reconstructing for the Future", Organization Science, vol. 18, n. 3, pp. 523-536.

GAVETTI G.M., HENDERSON R., GIORGI S. (2005), Kodak and The Digital Revolution (A), Harvard Business School Case 705-448, disponibile su www.hbs.edu.

HAECKEL S.H. (2004), "Peripheral vision: Sensing and acting on weak signals", Long Range Planning, vol. 37, n. 2, pp. 181-189.

HARRISON J.R., MARCH J.G. (1984), "Decision making and post decision surprises", Administrative Science Quarterly, vol. 29, n. 1, pp. 26-42.

HAUNSCHILD P.R., SULLIVAN B.N. (2002), "Learning from Complexity: Effects of Prior Accidents and Incidents on Airlines' Learning”, Administrative Science Quarterly, vol. 47, n. 4, pp. 609-646.

HERMANN C.F. (1972), "Threat, time and surprise”, in Hermann C.F. (a cura di), International Crises, Free Press, New York, pp. 187-211.

HUTCHINS E. (1995), Cognition in the Wild, MIT Press, Cambridge, MA.

JANIS I.L. (1982), Groupthink: Psychological Studies of Policy Decisions and Fiascoes, Houghton Mifflin, Boston, MA.

KENDRA J., WACHTENDORF T. (2006), “Community Innovation and Disasters”, in Rodríguez H., Quarantelli E.L., Dynes R.R. (a cura di), Handbook of Disaster Research, Springer New York, pp. 316-334.

KIESLER A., SPROULL L. (1982), "Managerial response to changing environments: perspectives on problem sensing from social cognition", Administrative Science Quarterly, vol. 27, n. 4, pp. 548-570.

KLEIN G., PLISKE R.M., CRANDALL B., WOODS D. (2005), "Problem detection”, Cognition, Technology, and Work, vol. 7, n. 4, pp. 14-28.

LAGADEC P. (2006), "Crisis Management in the Twenty-First Century "Unthinkable" Events in "Unthinkable" Contexts", in Rodriguez H., Quarantelli E.L., Dynes R.R. (a cura di), Handbook of Disaster Research, Springer New York, pp. 489-507. 
LAMPEL J.Z., SHAPIRA Z. (2001), "Judgmental errors, interactive norms, and the difficulty of detecting strategic surprises", Organization Science, vol. 12, n. 5, pp. 599-611.

LANZARA G.F. (1983), "Ephemeral organizations in extreme environments: Emergence, strategy, extinction", Journal of Management Studies, vol. 20, n. l, pp. 73-95.

LAPORTE T.R., CONSOLINI P.M. (1991), "Working in Practice but Not in Theory: Theoretical Challenges of High-Reliability Organizations", Journal of Public Administration Research and Theory, vol. 1, n. 1, pp. 19-48.

LEVITT B., MARCH J.G. (1988), "Organizational Learning", Annual Review of Sociology, vol. 14, pp. 319-338.

LYLES M.A., MITROFF I.I. (1980), “Organizational problem formulation: an empirical study”, Administrative Science Quarterly, vol. 25, n. 1, pp. 102-119.

MARCH J.G., SIMON H.A. (1958), Organizations, Blackwell Publishers, Cambridge, MA.

MARCH J.G., SPROULL L.S., TAMUZ M. (1991), "Learning from samples of one or fewer”, Organization Science, vol. 2, n. 1, pp. 1-13.

MARCUS A.A., NICHOLS M.L. (1999), "On the edge: Heeding the warning of unusual events", Organization Science, vol. 10, n. 4, pp. 482-499.

MINTZBERG H., RAISINGHANI D., THÉORÊT A. (1976), "The structure of "unstructured" decision processes", Administrative Science Quarterly, vol. 21, pp. 246-275.

MORRIS M., MOORE P. (2000), “The Lessons We (Don't) Learn: Counterfactual Thinking and Organizational Accountability after a Close Call", Administrative Science Quarterly, vol. 45, n. 5, pp. 737-765.

NARDUZZO A., WARGLIEN M. (1998), "Le routine come competenze organizzative", in Lipparini A. (a cura di), Competenze organizzative. Sviluppo, condivisione, trasferimento, Carocci, Roma.

NEWELL A., SIMON H.A (1972), Human Problem solving, Prentice Hall, Englewood Cliffs, New Jersey.

NELSON R.R., WINTER S.G. (1982), An evolutionary theory of economic change, Harvard University Press. Cambridge.

OCASIO W. (1997), "Towards an attention-based view of the firm", Strategic Management Journal, vol. 18, n. S1, pp. 187-206.

OLIVER D., ROOS J. (2003), "Dealing with the unexpected: Critical incidents in the LEGO Mindstorms team”, Human Relations, vol. 56, n. 9, pp. 1057-1082.

PERROW C. (1999), Normal Accidents: living with high-risk technologies, Princeton University Press, Princeton.

PERSICO F. (2014), "La gestione di un evento estremo come processo dinamico di coordinamento organizzativo", Studi Organizzativi, n. 2, pp. 9-41.

PINA e CUNHA M., CLEGG S.R., KAMOCHE K. (2006), "Surprises in Management and Organization: Concept, Sources and A Typology", British Journal of Management, vol. 17, n. 4, pp. 317-329.

QUARANTELLI E.L. (1988), "Disaster Crisis Management: A Summary of Research Findings", Journal of Management Studies, vol. 25, n. 4, pp. 373-385.

QUARANTELLI E.L., LAGADEC P., BOIN A. (2006), "A Heuristic Approach to Future Disasters and Crises: New, Old and In-Between Types", in Rodriguez H., Quarantelli H., Dynes R. (a cura di), Handbook of Disaster Research, Springer, pp. 16-41. 
sinergie Vol. 34, N. 99, 2016

RAMANUJAM R. (2003), "The effects of discontinuous change on latent errors in organizations: The moderating role of risk", Academy of Management Journal, vol. 46, n. 5, pp. 608-617.

RERUP C. (2009), "Attentional triangulation: Learning from unexpected rare crises", Organization Science, vol. 20, n. 5, pp. 876-893.

RUDOLPH J.W., REPENNING N.P. (2002), “Disaster Dynamics: Understanding the Role of Quantity in Organizational Collapse”, Administrative Science Quarterly, vol. 47, n. 1, pp. 1-30, doi:10.2307/3094889.

SCHÖN D. (1983), The Reflective Practitioner. How Professionals Think in Action, Temple Smith, London, UK.

SHACKLE G.L.S. (1979), Imagination and the Nature of Choice, Edinburgh University Press, Edinburgh, Ireland.

SEIDL D. (2004), "The Concept of Weak Signals Revisited: A Re-Description from a Constructivist Perspective", in Tsoukas H., Shepherd J. (a cura di), Managing the Future: Foresight in the Knowledge Economy, Blackwell, Oxford, pp. 151-168.

SHEAFFER Z., RICHARDSON B., ROSENBLATT Z. (1998), Early-warningsignals management: A lesson from the Barings crisis, vol. 6, n. 1, pp. 1-22.

SIMON H.A. (1947), Administrative behavior, The Free Press, New York, NY.

SIMON H.A. (1955), "A behavioral model of rational choice", The Quarterly Journal of Economics, vol. 69, n. 1, pp. 99-118.

SMITH G. (1989), "Defining Managerial Problems: A Framework for Prescriptive Theorizing", Management Science, vol. 35, n. 8, pp. 963-981.

STARBUCK W.H. (1983), "Organizations As Action Generators", American Sociological Review, vol. 48, n. 1, pp. 91-102.

SUCHMAN L.A. (1987), Plans and situated actions: The problem of humanmachine communications, Cambridge University, Cambridge, UK.

TALEB N. (2007), The Black swan: the Impact of the Highly Improbable, Random House, New York NY.

TAMUZ M. (2004), "Understanding accident precursors”, in Bier V.M., Kunreuther H., Phimister J.R. (a cura di), Accident precursor analysis and management: reducing technological risk through diligence, National Academies Press, Washington DC.

TIERNEY K.J., LINDELL M.K., PERRY R.W. (2001) Facing the unexpected: disaster preparedness and response in the United States, Joseph Henry Press, Washington DC.

THOMPSON K.D. (1967), Organization in Action, Mc Graw Hill, New York.

TRAINOR J.E. (2004), Searching For a System: Multi-Organizational Coordination in the September 11th World Trade Center Search and Rescue Response. Public Entity Risk Institute, University of Delaware, Disaster Research Center Preliminary Paper \#343.

TRIPSAS M., GAVETTI G. (2000), “Capabilities, Cognition, and Inertia: Evidence from Digital Imaging”, Strategic Management Journal, vol. 21, n. 10-11, pp. 1147-1161.

TURNER B.A. (1976), “The organizational and inter-organizational development of disasters", Administrative Science Quarterly, vol. 21, n. 3, pp. 378-397.

TURNER B.A, PIDGEON N. (1997), Man-Made disasters, ButterworthHeinemann. 
VAUGHAN D. (1996), The Challenger Launch Decision: Risky Technology, Culture, and Deviance at NASA, University Press, Chicago.

Maria Laura Frigotto Alessandro Narduzzo Sbiancare il cigno nero?

VON HAYEK F.A. (1937), "Economics and Knowledge", Economica, vol. 4, n. 13, Strategie e cor pp. 33-54.

WEICK K.E. (1990), "The Vulnerable System: An Analysis of the Tenerife Air Disaster", Journal of Management, vol. 16, n. 3, pp. 571-593.

WEICK K.E. (1993), "The Collapse of Sensemaking in Organizations: The Mann Gulch Disaster", Administrative Science Quarterly, vol. 38, n. 4, pp. 628-652.

WEICK K.E., SUTCLIFFE K.M. (2007), Managing the Unexpected, Jossey-Bass, San Francisco.

WEICK K.E., SUTCLIFFE K.M., OBSTFELD D. (1999), "Organizing for high reliability: Processes of collective mindfulness", in Sutton R., Staw B. (a cura di), Research in Organizational Behavior, JAI, Greenwich, CT, pp. 81- 124.

WINTER S. (2004), "Specialised perception, selection, and strategic surprise: learning from the moths and bees", Long Range Planning, vol. 37, n. 2, pp. 163-169, doi:10.1016/j.lrp.2004.01.002.

\section{Academic or professional position and contacts}

\section{Maria Laura Frigotto}

Researcher of Organization studies

University of Trento - Italy

e-mail: marialaura.frigotto@unitn.it

\section{Alessandro Narduzzo}

Full Professor of Management

Free University of Bozen - Bolzano - Italy

e-mail: anarduzzo@unibz.it 